\title{
Hard Parton Rescatterings and Minijets in Nuclear Collisions at the LHC
}

\author{
Alberto Accardi and Daniele Treleani] \\ Dipartimento di Fisica Teorica, Università di Trieste, \\ Strada Costiera 11, I-34014 Trieste \\ and \\ INFN, Sezione di Trieste \\ via Valerio 2, I-34127 Trieste
}

\begin{abstract}
The average number of minijets and the corresponding transverse energy produced in heavy ion collisions are evaluated by including explicitly semi-hard parton rescatterings in the dynamics of the interaction. At the LHC semi-hard rescatterings have a sizable effect on global characteristics of the typical inelastic event. An interesting feature is that the dependence on the cutoff which separates soft and hard parton interactions becomes less critical after taking rescatterings into account.
\end{abstract}

PACS: 11.80.La, 24.85.+p, 25.75.-q

\footnotetext{
${ }^{1}$ E-mail:accardi@ts.infn.it

${ }^{2}$ E-mail:daniel@ts.infn.it
} 


\section{Introduction}

Semi-hard physics is one of the most important issues in the interaction of heavy ions at the LHC. Given the total energy involved and the large number of participants, the component of the inelastic interaction which can be described within a perturbative approach is in fact rather substantial in heavy ion collisions at the LHC [1, 2]. The result is the production of a large number of minijets in the typical inelastic event. The description of the semihard component of the interaction, adopted by the majority of the papers on the subject, follows the approach of ref.|3]: the semi-hard component of the inelastic cross section, in a collision of two nuclei with atomic mass numbers $A$ and $B$, is written as:

$$
\begin{aligned}
\sigma_{H} & =\int d^{2} \beta\left(1-e^{-\sigma_{J} T_{A B}(\beta)}\right) \\
& =\sum_{n=1}^{\infty} \int d^{2} \beta \frac{\left(\sigma_{J} T_{A B}(\beta)\right)^{n}}{n !} e^{-\sigma_{J} T_{A B}(\beta)}
\end{aligned}
$$

where

$$
\begin{aligned}
\sigma_{J} & =\int_{x x^{\prime} s>4 p_{0}^{2}} d x d x^{\prime} \sum_{f f^{\prime}} G_{A}^{f}(x) \sigma^{f f^{\prime}}\left(x x^{\prime}\right) G_{B}^{f^{\prime}}\left(x^{\prime}\right) \\
T_{A B}(\beta) & =\int d^{2} b \tau_{A}(b) \tau_{B}(b-\beta)
\end{aligned}
$$

In Eq. (1.2) $\tau_{A}(b)$ and $\tau_{B}(b-\beta)$ are the thickness functions of the two interacting nuclei, normalized to $\mathrm{A}$ and $\mathrm{B}$, respectively, and depending on the transverse coordinates of the interacting partons $b$ and $b-\beta$, where $\beta$ is the impact parameter of the nuclear collision. $\sigma_{J}$ is the single scattering cross section to produce jets, expressed as a convolution of the nuclear parton distributions $G_{A}^{f}(x), G_{B}^{f^{\prime}}\left(x^{\prime}\right)$ and of the partonic cross section $\sigma^{f f^{\prime}}$ (integrated on the momentum transfer with the cutoff $p_{0}$ and without the multiplicity factor of the produced jets). The indices $f$ and $f^{\prime}$ label the different kinds of interacting partons and the momentum fractions $x, x^{\prime}$ are defined with respect to the single nucleon momentum. The dependence on the scale factor is implicit in all quantities.

The semi-hard cross section, as expressed in Eq.(1.1), represents the contribution to the total inelastic cross section of all events with at least one semi-hard partonic interaction, so that one may write $\sigma_{\text {inel }}=\sigma_{H}+\sigma_{\text {soft }}$, the two components being separated by means of $p_{0}$. Eq.(1.1) may be derived in an eikonal approach to nucleus-nucleus interactions, by writing the eikonal phase as the sum of a soft and of a hard component [1, 4. The physical picture corresponding to Eq.(1.1) is that of a distribution of multiple independent parton collisions localized in different points in transverse space and with the average number depending on the nuclear impact parameter. The average number of parton interactions, at fixed $\beta$, is $\langle N(\beta)\rangle=\sigma_{J} T_{A B}(\beta)$ so that, if $\sigma_{J}$ is at the lowest order in $\alpha_{S}$, the average multiplicity of minijets in a given nuclear collision (that is at $\beta$ fixed) is $2\langle N(\beta)\rangle$. The integrated inclusive cross section for producing minijets is therefore given by $2 \int d^{2} \beta\langle N(\beta)\rangle$. To obtain the inclusive cross section one needs, in fact, to count the jets produced with their multiplicity, so that, in the case of multiple parton interactions, the inclusive cross section is obtained by taking the average of the distribution in the number of parton collisions. The result is that the integrated inclusive cross section is 
given by the single scattering expression, $\sigma_{J}$, multiplied by the average multiplicity of jets produced in a single partonic interaction, which shows that the description of the process, as given by Eq.(1.1), is consistent with the AGK cancellation [5] (all unitarity corrections cancel in the inclusive). The cancellation property obviously holds for all averages, that are therefore equal to the result obtained by means of the single scattering expression, so that the transverse energy produced by minijets is given by

$$
\left\langle E_{t}(\beta)\right\rangle=2 T_{A B}(\beta) \int_{p_{t} \geq p_{0}} p_{t} \frac{d \sigma_{J}}{d^{2} p_{t}} d^{2} p_{t} .
$$

The semi-hard cross section $\sigma_{H}$ is a smooth function of $p_{0}$ for small values of the cut-off: the limiting value of $\sigma_{H}$ is in fact the geometrical limit $\pi\left(R_{A}+R_{B}\right)^{2}, R_{A}$ and $R_{B}$ being the two nuclear radii. On the contrary $\langle N(\beta)\rangle$ and $\left\langle E_{t}(\beta)\right\rangle$ are singular at small $p_{0}$ and their behaviour may be roughly estimated on dimensional grounds to be $\langle N(\beta)\rangle \simeq 1 / p_{0}^{2}$ and $\left\langle E_{t}(\beta)\right\rangle \simeq 1 / p_{0}$. The singular behavior at low $p_{0}$ can be used to set the limits of validity of the picture. Indeed, for the picture of the interaction to be valid one should take a relatively large value of the cut-off $p_{0}$; in this way the whole semi-hard interaction takes place in a relatively dilute system and the overall number of interactions will be relatively small. To deal with a regime where the number of parton interactions and the density of the interacting partons are large, the main modification adopted by the majority of papers is to include shadowing corrections in the nuclear parton distributions [6, 7]. In this way one obtains a substantial reduction of the number of projectile and target partons at low $x$ and the picture can be extended to sizably lower values of the cutoff $p_{0}$. Even so, when $p_{0}$ is further reduced, one reaches the condition of a highly dense interacting system where the whole picture ceases to be valid, which sets the lower limit for a sensible choice of the cutoff $p_{0}[8,9]$.

The overall resulting features are therefore that $p_{0}$ is different when varying the atomic mass number of the interacting nuclei and their energy, and that the distribution in the number of hard collisions at a fixed value of the impact parameter $\beta$ is a Poissonian in the whole semi-hard regime so that all average quantities are computed, as above, with the single scattering expression.

The clean physical interpretation of the approach, which incorporates the geometrical features of the nuclear process, unitarity, the factorization of the hard component of the interaction and the AGK cancellation rule, justifies the great success of the picture. Still there are a few delicate points which deserve further investigation and where the description of the process might be improved.

A very general approach to nucleus-nucleus interactions is trough the Reggeon Diagram Technique, where at high energies the interaction is described by the exchange of many Pomerons, including both independent exchanges between different nucleons and multi-Pomeron interactions, which represent the collision of a given projectile nucleon with several different target nucleons in a given interaction process. Each partonic collision corresponds to a fluctuation with a large transverse momentum inside the structure of an exchanged Pomeron. While the simplest case is that with a single partonic loop with large $p_{t}$, whose discontinuity corresponds to a $2 \rightarrow 2$ partonic collision, one might imagine more complicated fluctuations, with several connected parton lines, all with large $p_{t}$, in the structure of a multi-Pomeron interaction. The simplest case of this kind is 


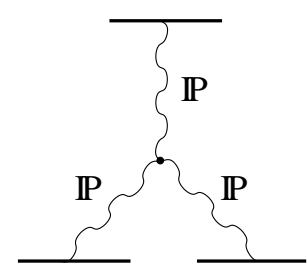

a)

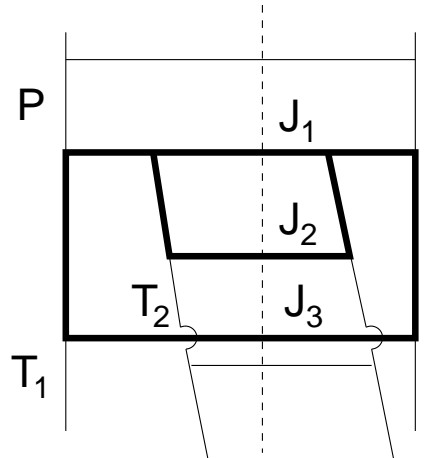

b)

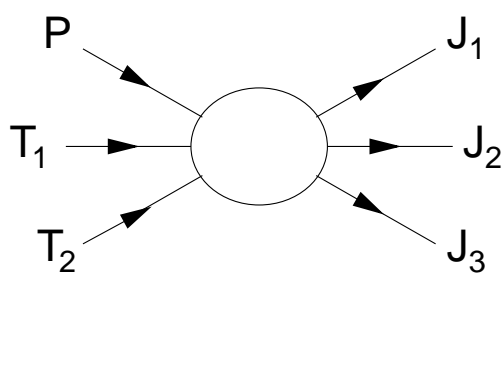

c)

Figure 1: a) A triple pomeron interaction between 3 nucleons. b) Parton ladders with the topology of Fig.11a). The thick lines are fluctuations with high $p_{t}$ and the dashed line is a possible cut. c) Partonic view of the cut of Fig. 1] $\mathrm{b}$ ): $\mathrm{P}$ is a projectile parton interacting with two target partons $\mathrm{T}_{i}$ and the final state is given by the three large- $p_{t}$ jets $\mathrm{J}_{i}$.

illustrated in Fig.1, where the Pomerons are represented by parton ladders. Discontinuities of such fluctuations originate configurations, where the compensation of transverse momenta in the final state involves several large $p_{t}$ partons, while the whole large $p_{t}$ configuration is generated by partons belonging to different chains, representing Pomerons attached to different nucleon lines. In the picture of the semi-hard interaction just recalled those configurations contribute to the shadowing corrections of the nuclear parton distributions. Their discontinuities, on the contrary, are not included in the semi-hard interaction dynamics. On the other hand, when approaching the black disk limit, the initial and final partonic states become locally isotropic in transverse space, so that both initial and final state configurations need to be built up with lots of partons. A proper discussion of semi-hard dynamics in the black disk limit requires therefore taking into account fluctuations with many large $p_{t}$ parton lines, which are to be included not only in the virtual corrections but also in the production process. Hence one needs to consider partonic interactions where several partons, with low virtuality and $p_{t}$ and sizable longitudinal components (so that, in the nucleus-nucleus c.m. frame, each one may be ascribed to a definite nucleus) interact producing large $p_{t}$ jets. The simplest possibility of this kind (one projectile against two targets, $t / s \rightarrow 0$ and basically electro-dynamical interaction between point like objects) was discussed in Ref. [10], where the forward amplitude of the process and all the cuts were derived. The final result of the analysis was that, in the $t / s \rightarrow 0$ limit, the different cuts of the amplitude are all proportional one to another, the proportionality factors being the AGK weights. It was moreover possible to express the three body interaction as a product of two body interaction probabilities. Therefore, one may argue that, in the black disk limit and in the nucleus-nucleus c.m. frame, a projectile parton interacts with all the various nearby partons of the target in the different directions in transverse space, and that the corresponding cross section may be approximated by the product of two-body interaction cross sections, so that one might call such a process re-interaction or re-scattering.

An attempt to introduce such more elaborate semi-hard dynamics, including explicitly semi-hard parton rescatterings in the interaction, was done in ref. [11] and [12]. Both the 
average number of minijets and the average transverse energy are modified by semi-hard rescatterings, and an interesting feature is that both quantities develop a less singular dependence on the cutoff, in such a way that the choice of $p_{0}$ becomes less critical when semi-hard parton rescatterings are taken into account. The average number of minijets and the transverse energy produced in heavy ion collisions have been recently discussed in several papers, with the purpose to determine the initial conditions for the further evolution and termalization of the system (see e.g. [7, 13]). We think that it might be interesting to have an indication of the effects of rescatterings on these quantities at the LHC, and we'll discuss this topic in the present paper. In the next section, after including rescatterings in the picture of the interaction, we'll recall the expression of the average number of minijets and derive the corresponding average transverse energy. Then, we'll give some quantitative indication on the effect and comment the qualitative features induced by the more structured interaction dynamics.

\section{Multiple Parton Scatterings and Average Number of Minijets}

The introduction of semi-hard parton rescatterings can be obtained in a picture of the interaction where the soft part is factorized in a Poissonian multi-parton distribution and the hard part is expressed in terms of perturbative parton-parton collisions. The nuclear multi-parton distributions are unknown quantities and the reason of the choice of the Poisson distribution is that it corresponds to the case where the information on the initial state is minimal, since the whole distribution is expressed in terms of its average value only. One has moreover the possibility of introducing systematically further informations on the nuclear partonic structure in terms of correlations among partons [14]. The hard part is written in terms of two-body collisions by introducing the probability of having at least one interaction between the two configuration, in a way analogous to the expression of the inelastic nucleus-nucleus cross section [15]. The process is therefore represented as the sum of all possible interactions between all configurations with a definite number of partons of the two nuclei. In this way the semi-hard component of the cross section can be written as

$$
\begin{aligned}
\sigma_{H}=\int d^{2} \beta & \sum_{n=1}^{\infty} \frac{1}{n !} \Gamma_{A}\left(x_{1}, b_{1}-\beta\right) \ldots \Gamma_{A}\left(x_{n}, b_{n}-\beta\right) e^{-\int d x d^{2} b \Gamma_{A}(x, b-\beta)} \\
& \cdot \sum_{l=1}^{\infty} \frac{1}{l !} \Gamma_{B}\left(x_{1}^{\prime}, b_{1}^{\prime}\right) \ldots \Gamma_{B}\left(x_{l}^{\prime}, b_{l}^{\prime}\right) e^{-\int d x^{\prime} d^{2} b^{\prime} \Gamma_{B}\left(x^{\prime}, b^{\prime}\right)} \\
\cdot & {\left[1-\prod_{i=1}^{n} \prod_{j=1}^{l}\left(1-\hat{\sigma}_{i j}\right)\right] d x_{1} d^{2} b_{1} \ldots d x_{n} d^{2} b_{n} d x_{1}^{\prime} d^{2} b_{1}^{\prime} \ldots d x_{l}^{\prime} d^{2} b_{l}^{\prime} }
\end{aligned}
$$

where

$$
\Gamma_{A}(x, b)=\tau_{A}(b) G(x)
$$


To keep the notation as simple as possible, the indices labeling the different kinds of partons have been suppressed and the dependence on the cutoff $p_{0}$ is implicit. The two Poissonian distributions, with average numbers $\Gamma_{A}(x, b-\beta)$ and $\Gamma_{B}\left(x^{\prime}, b^{\prime}\right)$, represent the multi-parton distributions of the two interacting nuclei. The probability to have at least one partonic interaction, given a configuration with $n$ and $l$ partons in the two nuclei, is represented by the square parenthesis in Eq.(2.1) and, according with the discussion in the previous paragraph, is constructed by means of the probability $\hat{\sigma}_{i j}$ of interaction of a given pair of partons $i$ and $j$. The interaction probability is a function of the cutoff $p_{0}$, so that only the interactions with momentum exchange larger than $p_{0}$ are taken into account in (2.1). The majority of interactions takes place with a momentum exchange close to the cutoff value, we therefore evaluate the parton distributions at the scale of the cutoff. Since the distance over which the hard interactions are localized is much smaller than the typical nuclear radius, the probability of interaction can be approximated by $\hat{\sigma}\left(x_{i} x_{j}, b_{i}-b_{j}\right)=\sigma\left(x_{i} x_{j}\right) \delta^{(2)}\left(b_{i}-b_{j}\right)$.

The most important features of Eq.(2.1) are that all possible interactions between the two partonic configurations are included, and that probability conservation is explicitly taken into account by the term in square parentheses. The Eikonal cross-section in Eq.(1.1) is obtained when one neglects all rescatterings in Eq.(2.1), in such a way that each parton is allowed to interact at most once [16]. If, on the contrary, one keeps rescatterings into account one cannot write a closed expression for $\sigma_{H}$. However, it is possible to obtain simple expressions from Eq.(2.1) for many relevant quantities.

If one works out the average number of parton collisions $\langle N(\beta)\rangle$ one obtains, as in the traditional approach, the single scattering expression $\langle N(\beta)\rangle=\sigma_{J} T_{A B}(\beta)$ [11]. So the overall average number of parton collisions satisfies the AGK cancellation and is not affected by any unitarity correction; however it is not a simple quantity to observe. A more directly observable quantity, or at least one which can be more directly related to observable quantities, is the average number of produced minijets. An important effect of including rescatterings is that the number of produced minijets is no more proportional to the number of collisions, because now each projectile parton is allowed to interact more than once with the target. As a consequence, while unitarity corrections do not change the average number of collisions, they affect the average number of minijets produced in the nuclear collision.

The correction term can be derived in a straightforward way from Eq.(2.1) [1], but one may use also a more direct argument. One may in fact obtain the usual semi-hard cross section, Eq.(1.1), by starting from the single scattering cross section to produce large- $p_{t}$ jets. In the perturbative QCD-parton model the cross section is written as:

$$
\sigma_{J}=\int_{x x^{\prime} s>4 p_{0}} d^{2} \beta d^{2} b d x d x^{\prime} \Gamma_{A}(x, b-\beta) \sigma\left(x x^{\prime}\right) \Gamma_{B}\left(x^{\prime}, b\right)=\int d^{2} \beta\langle N(\beta)\rangle .
$$

The expression needs however to be unitarized also when the cutoff $p_{0}$ has a rather large value, since $\sigma_{J}$ is proportional to the large factor $A \times B$. Eq.(1.1) is in fact the result of the $s$-channel unitarization of $\sigma_{J}$ and may be obtained by noticing that in Eq. $(2.2)\langle N(\beta)\rangle$ is dimensionless and may be understood as the average number of parton interactions at a given impact parameter $\beta$. The $s$-channel unitarized cross section $\sigma_{H}$ is the result of replacing this average number with the interaction probability, which, if the distribution in the number of interactions is a Poissonian, is just $1-\exp (-\langle N(\beta)\rangle)$. Hence the unitarized 
cross section $\sigma_{H}$ represents the contribution to the total cross section of all events with at least one couple of partons interacting with a transverse momentum exchange above $p_{0}$, as it is clear from the second line of Eq.(1.1). On the other hand $\sigma_{J}$, that includes also the multiplicity of the interactions, represents rather the integrated inclusive cross section (apart from the factor representing the average multiplicity of jets produced in a single collision).

When the cutoff is moved towards low values and rescatterings need to be taken into account, the average number of jets produced is no more proportional to the average number of collisions. In this case one may proceed by applying to $\langle N(\beta)\rangle$ an argument analogous to that previously used to unitarize $\sigma_{J}$. By looking at Eq.(2.2) one can identify

$$
\left\langle n_{B}(x, b)\right\rangle \equiv \int_{x x^{\prime} s>4 p_{0}^{2}} d x^{\prime} \Gamma_{B}\left(x^{\prime}, b\right) \sigma\left(x x^{\prime}\right)
$$

as the average number of collisions of each interacting A-parton at fixed $x$ and $b$. Then one can write the average number of produced minijets at fixed impact parameter as

$$
2\langle N(\beta)\rangle=\int_{x s>4 p_{0}^{2}} d^{2} b d x \Gamma_{A}(x, b-\beta)\left\langle n_{B}(x, b)\right\rangle+\int_{x^{\prime} s>4 p_{0}^{2}} d^{2} b d x^{\prime} \Gamma_{B}\left(x^{\prime}, b\right)\left\langle n_{A}\left(x^{\prime}, b-\beta\right)\right\rangle,
$$

that represents the average number of incoming partons from the nucleus A multiplied by the average number of collisions against the partons of nucleus $B$, plus the analogous term with $\mathrm{A}$ and $\mathrm{B}$ interchanged. Then, if one replaces in Eq.(2.4) the average number of scatterings of each parton with its interaction probability, viz. $1-\exp \left(\left\langle n_{B}(x, b)\right\rangle\right)$ and $1-\exp \left(\left\langle n_{A}(x, b-\beta)\right\rangle\right)$, one obtains the average number of "wounded partons" $W_{A}(x, b)$ and $W_{B}\left(x^{\prime}, b-\beta\right)$ of the two nuclei. These ones are the partons of the two nuclei that had at least one hard interaction. The expression of the average number of wounded partons of nucleus $A$ (with transverse coordinate $b$ and momentum fraction $x$, in an event with nuclear impact parameter $\beta$ ) is therefore

$$
W_{A}(x, b, \beta)=\Gamma_{A}(x, b-\beta)\left[1-e^{-\left\langle n_{B}(x, b)\right\rangle}\right] .
$$

Every wounded parton obtained in this way produces a minijet in the final state and the transverse energy produced by semi-hard interactions is the transverse energy carried by the wounded partons. As a consequence both the average number of minijets and their average transverse energy are quantities affected by the presence of rescatterings and the corresponding correction term is more and more important when the average number of scatterings $\left\langle n_{B}\right\rangle$ becomes larger and larger, namely at low values of the cutoff $p_{0}$ and (or) for large atomic mass numbers.

The overall number of produced minijets, i.e. the sum of the wounded partons of nucleus $A$ with those of nucleus $B$, obviously coincides with the usual result $2\langle N(\beta)\rangle$, when the average number of rescatterings is small. When the number of rescatterings is large the two quantities are however different and, while the average number of collisions $\langle N(\beta)\rangle$ may be divergent in the saturation limit, the average number of wounded partons is on the contrary well defined. In fact one obtains that the square parenthesis in Eq. (2.5) has 1 as a limiting value and, in this limit, the average number of wounded partons is just the sum of the average number of partons of the two interacting nuclei. 

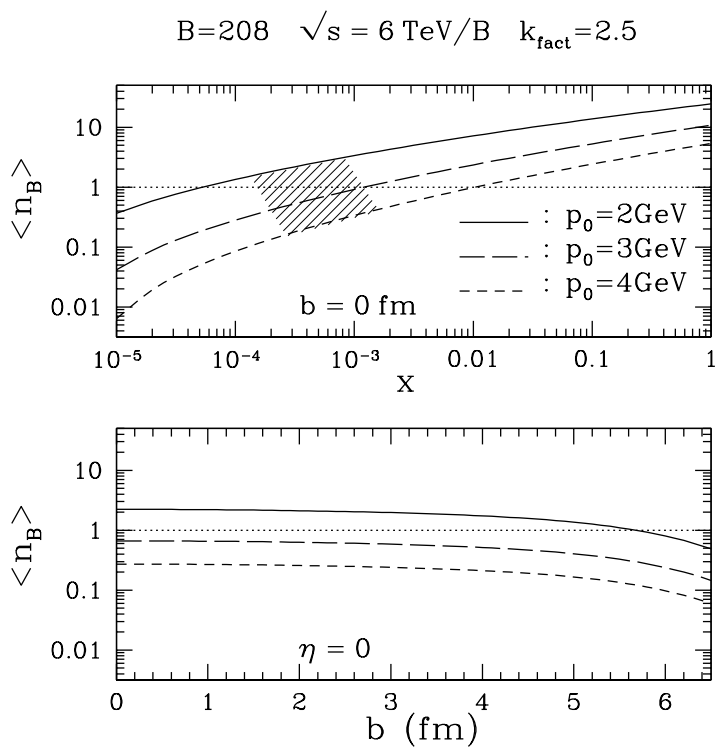

Figure 2: Average number $\left\langle n_{B}\right\rangle$ of semi-hard scatterings suffered by a parton from the A-nucleus while it is interacting with the $\mathrm{B}$-nucleus. We consider a $\mathrm{Pb}-\mathrm{Pb}$ collision at a c.m. energy $\sqrt{s}=6 \mathrm{TeV} / \mathrm{A}$, with a scale $Q=p_{0} / 2$ and a $k$-factor $k=2.5$; Woods-Saxon thickness functions are used. Top: $\left\langle n_{B}\right\rangle$ is plotted as a function of the projectile parton fractional momentum $x$ in the case of a central parton-nucleus collision. The three curves are the result for a cut-off $p_{0}=2 \mathrm{GeV}$ (solid line), $3 \mathrm{GeV}$ (long dashes), 4 $\mathrm{GeV}$ (short dashes). The shaded area corresponds to the central pseudo-rapidity region $|\eta| \leq .9$, where $\eta=\log \left(x \sqrt{(s)} / p_{0}\right)$. The dotted line marks the transition value of 1 scattering per parton. Bottom: $\left\langle n_{B}\right\rangle$ is plotted as a function of the impact parameter for a parton emerging with pseudo-rapidity $\eta=0$.

To have a quantitative feeling for the regime of interest at LHC energies we plot $\left\langle n_{B}\right\rangle$, the average number of scatterings per parton from the A-nucleus, as a function of the projectile parton fractional momentum $x$ and impact parameter $b$. As one can see, at a cut-off $p_{0}=2 \mathrm{GeV}$ a parton in the central region experiences on average 1 to 3 scatterings over the whole target transverse area except the very peripheral regions. As discussed above, at higher cut-off values the number of scatterings decreases. As a result, semi-hard rescatterings should be negligible down to $p_{0} \simeq 3 \mathrm{GeV}$ where their effects begin to show up, and already at $p_{0}=2 \mathrm{GeV}$ they should have a large effect and should not be neglected. These conclusions will be confirmed by the numerical computations discussed in section 田.

In summary, the effect of rescatterings on the average number of the produced minijets is to reduce the number obtained by means of the single scattering expression, not differently, qualitatively, from the result of including shadowing corrections in the nuclear parton distribution. On the other hand the overall distribution in the number of minijets produced is modified. In the traditional approach there is a strong correlation in the distribution of the number of minijets, since each minijet has a recoiling companion; when the average number of rescatterings increases this correlation gets weaker and weaker, so that in the high density limit no correlation is left and the distribution tends to a Poissonian [11]. A further important difference is that, since $W_{A}$ and $W_{B}$ are well defined in this limit, after including rescatterings the average number of minijets becomes much less dependent on the choice of the cutoff at low $p_{0}$. 


\section{Average Transverse Energy}

As we have discussed in the last section, the average number of minijets is not the only quantity modified by this more elaborate interaction dynamics, which in fact has a nontrivial effect also on the transverse energy carried by the minijets 12 . The wounded partons of nucleus $A$ at a given $x$ and $b$ and in a nuclear interaction with impact parameter $\beta$, Eqn.(2.5) are obtained by multiplying the average number of partons of $A$ (with given $x$ and $b$ ) by the corresponding interaction probability, which is a Poisson probability distribution in the number of scatterings, with average $\left\langle n_{B}(x, b)\right\rangle=\int d x^{\prime} \Gamma_{B}\left(x^{\prime}, b\right) \sigma\left(x x^{\prime}\right)$ :

$$
W_{A}(x, b, \beta)=\Gamma_{A}(x, b-\beta) \sum_{\nu=1}^{\infty} \frac{\left\langle n_{B}(x, b)\right\rangle}{\nu !} e^{-\left\langle n_{B}(x, b)\right\rangle} .
$$

We may therefore obtain the differential distribution in the transverse momentum $p_{t}$ of the wounded $A$-partons by introducing a constraint in the transverse momentum intergrals that give the total cross sections in the above expression:

$$
\begin{aligned}
& \frac{d W_{A}}{d^{2} p_{t}}(x, b, \beta)= \\
& =\Gamma_{A}(x, b-\beta) \sum_{\nu=1}^{\infty} \frac{1}{\nu !} \int \Gamma_{B}\left(x_{1}^{\prime}, b\right) \ldots \Gamma_{B}\left(x_{\nu}^{\prime}, b\right) e^{-\int d x^{\prime} \Gamma_{B}\left(x^{\prime}, b\right) \sigma\left(x x^{\prime}\right)} \\
& \quad \cdot \frac{d \sigma}{d^{2} k_{1}} \ldots \frac{d \sigma}{d^{2} k_{\nu}} \delta^{(2)}\left(\mathbf{k}_{1}+\ldots+\mathbf{k}_{\nu}-\mathbf{p}_{\mathbf{t}}\right) d^{2} k_{1} \ldots d^{2} k_{\nu} d x_{1}^{\prime} \ldots d x_{\nu}^{\prime}
\end{aligned}
$$

The limits of integration on $x_{i}^{\prime}$ and $x^{\prime}$ are respectively $x x_{i}^{\prime} s \geq 4 k_{i}^{2}$ and $x x^{\prime} s \geq 4 p_{0}^{2}$, and all the distribution functions are evaluated for simplicity at a fixed scale. To obtain the corresponding average transverse energy $\left\langle p_{t}(x, b, \beta)\right\rangle_{A}$ one has to integrate Eq.(3.2) with an additional factor $p_{t}$. A convenient way to proceed is to introduce the Fourier transform of the parton-parton scattering cross section

$$
\tilde{\sigma}(u)=\int d^{2} k e^{i \mathbf{k} \cdot \mathbf{u}} \frac{d \sigma}{d^{2} k} \quad ; \quad \tilde{\sigma}(0)=\sigma
$$

and to express the $\delta$-function in Eq.(3.2) in an integral form. Since $\tilde{\sigma}(u)$ depends only on the modulus of $\mathbf{u}$ one obtains

$$
\begin{aligned}
\left\langle p_{t}(x, b, \beta)\right\rangle_{A}= & \Gamma_{A}(x, b-\beta) \int_{0}^{\infty} d p_{t} p_{t}^{2} \int_{0}^{\infty} d u u J_{0}\left(p_{t} u\right) \cdot \\
& \cdot \sum_{\nu=1}^{\infty} \frac{1}{\nu !}\left[\int d x^{\prime} \Gamma_{B}\left(x^{\prime}, b\right) \tilde{\sigma}(u)\right]^{\nu} e^{-\int d x^{\prime} \Gamma_{B}\left(x^{\prime}, b\right) \tilde{\sigma}(0) .}
\end{aligned}
$$

After multiplying the integrand by the exponential factor $\exp \left(-\lambda p_{t}\right)$ one can exchange the two integrals and first do the integral on $p_{t}$. The result of this integration is

$$
\int_{0}^{\infty} e^{-\lambda p_{t}} J_{0}\left(p_{t} u\right) p_{t}^{2} d p_{t}=\frac{2 \lambda^{2}-u^{2}}{\left(\lambda^{2}+u^{2}\right)^{5 / 2}}=\frac{1}{u} \frac{d}{d u}\left[\frac{\lambda^{2} u^{2}+u^{4}}{\left(\lambda^{2}+u^{2}\right)^{5 / 2}}\right],
$$

which allows one to perform the integral on $u$ by parts. After differentiating the series in $\nu$ one obtains the factor

$$
\tilde{\sigma}^{\prime}(u)=-2 \pi \int_{0}^{\infty} k^{2} J_{1}(k u) \frac{d \sigma}{d^{2} k} d k
$$


which is proportional to $u$ because of the argument of the $J_{1}$ Bessel function. The integrand in $u$ is therefore regular for $u=0$ also in the limit $\lambda \rightarrow 0$. Then one takes this limit and sums the series in $\nu$. The final expression has a closed analytical form:

$$
\left\langle p_{t}(x, b, \beta)\right\rangle=-\Gamma_{A}(x, b-\beta) \int_{0}^{\infty} d u d x^{\prime} \frac{\tilde{\sigma}^{\prime}(u)}{u} \Gamma_{B}\left(x^{\prime}, b\right) e^{\int d x^{\prime} \Gamma_{B}\left(x^{\prime}, b\right)[\tilde{\sigma}(u)-\tilde{\sigma}(0)]}
$$

The average transverse energy in an event with a given impact parameter $\beta$ is the result of integrating Eq.(3.7) on $b$ and $x$ and of summing the two contributions of the wounded partons of the nuclei $A$ and $B$. Notice that the expression in Eq.(3.7) is much less dependent on the choice of the cutoff $p_{0}$ than the usual average energy evaluated with the single scattering expression of the perturbative QCD parton model: the Rutherford singularity of the parton-parton cross section is in fact smoothed in Eq.(3.6) by the Bessel function $J_{1}(k u)$, in such a way that the dependence on the cutoff $p_{0}$ is only logarithmic. The same logarithmic dependence on the cutoff is present in the argument of the exponential

$$
\tilde{\sigma}(u)-\tilde{\sigma}(0)=2 \pi \int\left[J_{0}(k u)-1\right] \frac{d \sigma}{d^{2} k} k d k
$$

as a consequence of the behavior of $\left[J_{0}(k u)-1\right]$ for $k \rightarrow 0$.

In summary, when the semi-hard cross section is expressed by Eq.(2.1) one may obtain, without further approximations, a closed analytic form both for the average number of minijets and for the corresponding average transverse energy and in both cases the singular dependence for small values of the cutoff is smoothened by rescatterings.

\section{Discussion}

Many papers have been recently devoted to the production of minijets in heavy ion collisions [2, 7, 9, 13]. A rather general feature is that, because of the singular behavior of the elementary parton interaction at low momentum transfer, many relevant quantities depend rather strongly (typically like an inverse power) on the value of the cutoff which distinguishes soft and hard parton interactions. The feature is unpleasant since although one might find physical arguments to determine a meaningful value of the cutoff [8, 9, 17], it is rather difficult to fix it in a very precise way. We have therefore tried, in the present paper, to face this issue by studying the effect of a more elaborate interaction dynamics on the average number of minijets produced in a nuclear collision and on the corresponding average transverse energy. While in the traditional picture of the semi-hard processes each parton is allowed to interact with large momentum transfer only once, we have included semi-hard parton rescatterings in the dynamics of the interaction. Semi-hard rescatterings, that are negligible when the threshold between hard and soft processes is high, become more and more important when the threshold is lowered and the target approaches the black disk limit. Naively one would expect that the inclusion of rescatterings in the picture of the interaction might worsen the divergent behavior at low transverse momenta; on the contrary a more careful analysis, that takes probability conservation consistently into account, shows that the result is just the opposite. Following [11] and [12] we have in fact represented the semi-hard nuclear cross section with Eq.(2.1), 
where all possible multi-parton collisions, including rescatterings, are taken into account and the conservation of probability is explicitly implemented. The average number of minijets and the corresponding transverse energy, at fixed $x, b$ and impact parameter $\beta$, are then expressed in a closed analytic form by (2.5) and (3.7), whose behavior with the cutoff is much less singular in comparison with the analogous averages obtained without taking rescatterings into account. The reason of this smoother behavior is that rescatterings introduce (through $\Gamma_{A}$ and $\Gamma_{B}$ ) a new dimensional quantity in (2.5) and (3.7), the nuclear radius, which gives the dimensionality to the two average quantities at small $p_{0}$. When rescatterings are neglected the dimensionality at small $p_{0}$ is provided by the cutoff itself, and the result is that the two quantities behave as an inverse power of the cutoff for $p_{0} \rightarrow 0$.

Apart from the approximation of writing all connected multi-parton processes as products of $2 \rightarrow 2$ partonic collisions, our approach states on the assumption of neglecting production processes at the partonic level (namely $2 \rightarrow 3$ etc. parton processes) and of using forward kinematics in the nucleon-nucleon c.m. frame. To have a feeling on the validity of such approximations at LHC energy, we have evaluated the average energy of a partonic interaction in the parton-parton center of mass frame, and the average value of momentum fraction $x$ of a projectile parton:

$$
\begin{aligned}
\left\langle E_{c . m .}\right\rangle \sigma_{J} & =\int d x d x^{\prime} \sqrt{x x^{\prime} s} \sum_{f f^{\prime}} G_{A}^{f}(x) \sigma^{f f^{\prime}}\left(x x^{\prime}\right) G_{B}^{f^{\prime}}\left(x^{\prime}\right) \\
\langle x\rangle \sigma_{J} & =\int d x d x^{\prime} x \sum_{f f^{\prime}} G_{A}^{f}(x) \sigma^{f f^{\prime}}\left(x x^{\prime}\right) G_{B}^{f^{\prime}}\left(x^{\prime}\right)
\end{aligned}
$$

When the whole rapidity range is considered typical values are $\left\langle E_{\text {c.m. }}\right\rangle \approx 25 \mathrm{GeV},\langle x\rangle \approx$ $3 \times 10^{-2}$ (corresponding to a momentum of $\approx 45 \mathrm{GeV}$, if the nucleon-nucleon c.m. energy is $6 \mathrm{TeV})$ with $p_{0}=2 \mathrm{GeV}$. The average $\langle x\rangle$ becomes substantially smaller when averaging in a narrow window in the central rapidity region. The relatively low value of $\left\langle E_{c . m}.\right\rangle$, as compared with the cutoff, indicates that the inclusion of $2 \rightarrow 3$, or of higher order partonic processes, should not spoil the whole approach, that could therefore represent a reasonable lowest order approximation. The relatively large value of the momentum boost to go from the nucleon-nucleon c.m. frame to the partonic c.m. frame shows, on the other hand, that forward kinematics is reasonable in the former frame of reference.

The effect of semi-hard parton rescatterings on the average number of minijets and on the average transverse energy produced in a central $\mathrm{Pb}-\mathrm{Pb}$ collision at $\mathrm{LHC}$ energies is summarized in Fig.3. We plotted the average number of minijets and their transverse energy in the case of a very central rapidity window, $|y|<.9$, corresponding to the ALICE detector, and in a larger rapidity interval, $|y|<4$, that will be covered by the CMS detector. Figure $3 a$ ) shows, in the two cases, the dependence of the average number of produced minijets on the choice of the cutoff $p_{0}$. The dashed curves are the results obtained by the single scattering expression, Eq.(2.2), while the continuous curves are the result of the inclusion of semi-hard parton rescatterings, Eq.(2.5) plus the analogous term for the $B$-partons. These expressions have been computed with the GRV98LO distribution functions [18 with no shadowing corrections included, and by representing the elementary partonic interaction at the lowest order in QCD; to account for higher order corrections the result of the elementary interaction has been multiplied by a factor 

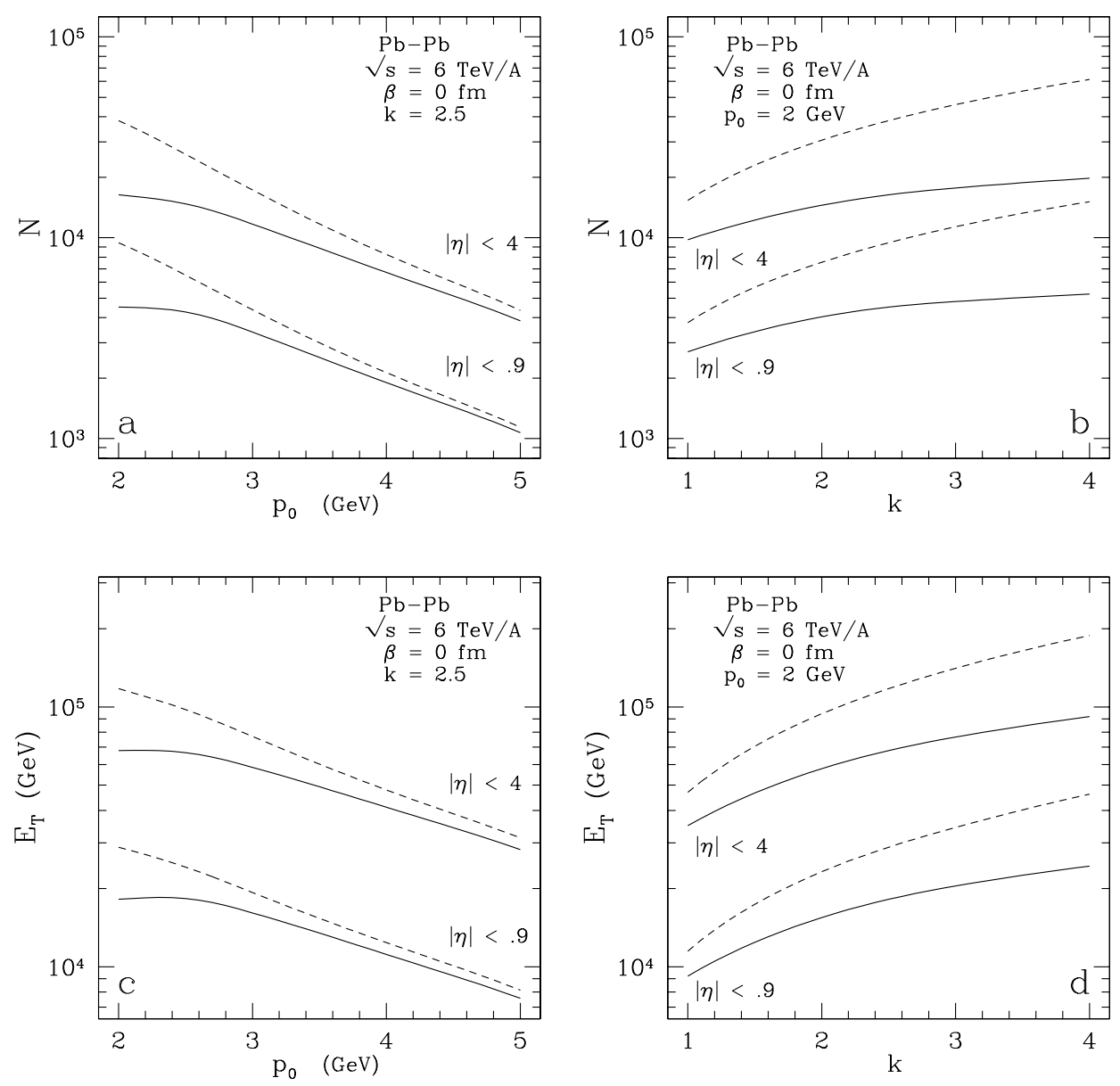

Figure 3: Average number $N$ of minijets, a) b), and average transverse energy $\left.E_{T}, c\right) d$ ), in a $\mathrm{Pb}-\mathrm{Pb}$ central collision in two different rapidity windows, $|y|<4$ and $|y|<.9$, as a function of the cutoff, $a$ ) and $c$ ), and of the $k$-factor, $b$ ) and $d$ ). The dashed curves are computed without including rescatterings, the continuous curves after including rescatterings.

$k=2.5$. Both the $k$-factor and the scale $Q=p_{0} / 2$ where chosen in order to reproduce the value of the $p \bar{p}$ mini-jet cross-section at $\sqrt{s}=900 \mathrm{GeV}$ [19]. The sensitivity to the value of the $k$-factor is shown in Fig. $3 b$ ), where the curves have the same meaning as in Fig. [3a), and the cutoff has been fixed to the value $p_{0}=2 \mathrm{GeV}$. Analogous curves for the average transverse energy carried by the produced minijets are shown in Fig. 3ic) and 3 $d$ ). The average transverse energy without rescatterings has been computed by using Eq.(1.3) (dashed curves) and with rescatterings (continuous curves) by using Eq.(3.7), after integrating on $b$ and on $x$ (inside the corresponding rapidity windows) and adding the analogous contribution of the $B$-partons. 


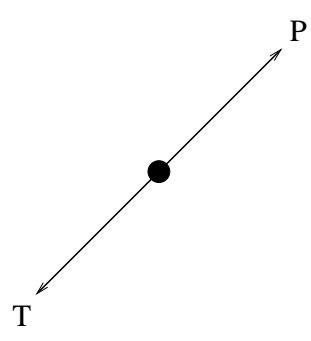

a)

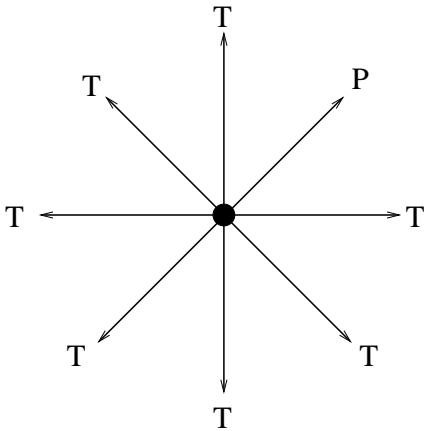

b)

Figure 4: Production of minijets in the transverse momentum plane. a) The projectile parton (P) is allowed to interact only once with a target parton $(\mathrm{T})$, so that the two produced minijets are back-to-back.

b) The projectile can scatter against more than one target and minijets are produced with a star-shape.

\section{Conclusions}

In this paper we have discussed the production of minijets in heavy ion collisions at the LHC, by including explicitly semi-hard parton rescatterings in the dynamics of the interaction. The regime of interest is, in fact, the regime where the nuclear target reaches the black disk limit for a projectile parton interacting with momentum exchange above the cutoff $p_{0}$ (which defines the lower limit in $p_{t}$ for minijet production). In the mechanism of production of minijets usually considered (a projectile parton interacts with only a single target parton) the elementary interaction generates a state with a preferred direction in transverse space (the direction of the two minijets) which is not consistent with local symmetry in the transverse directions implied by the black disk limit. The star-like shape in transverse space of the state produced by the multiple interactions of a projectile with different target partons (scattered projectile + recoils) recovers, on the other hand, the symmetry property of the interaction in the black disk limit, see Fig. $⿴$.

The basic element in our estimate is to recognize that the usual expression that gives the average number, $\langle N(\beta)\rangle$, of parton collisions at a fixed value of the nuclear impact paramete, Eq.(2.2), is obtained by convoluting the average number of partons of the projectile with the average number, $\left\langle n_{B}(x, b)\right\rangle$, of interactions of each projectile parton with the target nucleus, Eq.(2.3). Notice that the evaluation of $\left\langle n_{B}(x, b)\right\rangle$ and its dependence on the momentum fraction is, in this way, determined in a unique way by the parton distributions. Our results are therefore parameter free. Given the expression of the semi-hard cross section, Eq.(2.1), the average number of produced minijets, $W(x, b, \beta)$, Eq.(2.5), and the corresponding average transverse energy $\left\langle p_{t}(x, b, \beta)\right\rangle$, (Eq.3.7), are computed without further approximations, so that our result for $N$ and $E_{T}$, plotted in the figures, are exact consequences of the nuclear cross section (2.1). An approximation that is done when writing the nuclear cross section (2.1), is to evaluate the parton distributions at the scale of the cutoff, so that in Eq.(3.2) the only dependence on the transverse momenta is in the elementary partonic cross sections, which basically corresponds to neglecting the logarithmic dependence of the distributions in comparison with the inverse power dependence of the cross section. However, our formalism can be extended to take into account a general 
scale dependence.

The main features of the numerical evaluation are that semi-hard rescatterings have a sizable effect on the average number of minijets and on the transverse energy produced in heavy ion collisions at the LHC, so that they affect also global characteristics of the typical inelastic event. The induced correction increases with the value of the $k$-factor, which represents higher orders in the elementary parton collision, and with the size of the rapidity window, since rescatterings are more frequent for partons with a larger momentum fraction. By looking at the dependence on the cutoff, both the average number of minijets and the corresponding average transverse energy are more regular at low $p_{0}$, showing a tendency to saturate below $p_{0} \simeq 3 \mathrm{GeV}$ and making in this way the choice of the cutoff less critical.

\section{Acknowledgment}

This work was partially supported by the Italian Ministry of University and of Scientific and Technological Researches (MURST) by the Grant COFIN99. 


\section{References}

[1] Xin-Nian Wang, Phys. Rept. 280 (1997) 287;

[2] K. Kajantie, Nucl. Phys. A663, 191 (2000); N. Armesto and C. Pajares, Int. J. Mod. Phys. A15, 2019 (2000);

[3] K. Kajantie, P. V. Landshoff and J. Lindfors, Phys. Rev. Lett. 59, 2527 (1987);

[4] A. Capella, J. Tran Thanh Van and J. Kwiecinski, Phys. Rev. Lett. 58, 2015 (1987);

[5] V. A. Abramovsky, V. N. Gribov and O. V. Kancheli, Yad. Fiz. 18, 595 (1973); L. Bertocchi and D. Treleani, J. Phys. G G3, 147 (1977);

[6] A. H. Mueller and J. Qiu, Nucl. Phys. B268, 427 (1986); K. J. Eskola, J. Qiu and X. Wang, Phys. Rev. Lett. 72, 36 (1994); J. Jalilian-Marian and X. Wang, hep$\mathrm{ph} / 0005071$;

[7] V. Emel'yanov, A. Khodinov, S. R. Klein and R. Vogt, Phys. Rev. C61, 044904 (2000); N. Hammon, H. Stocker and W. Greiner, Phys. Rev. C61, 014901 (2000);

[8] L. V. Gribov, E. M. Levin and M. G. Ryskin, Phys. Lett. B121, 65 (1983); J. P. Blaizot and A. H. Mueller, Nucl. Phys. B289 (1987) 847;

[9] K. J. Eskola and K. Kajantie, Z. Phys. C75, 515 (1997); K. J. Eskola, K. Kajantie, P. V. Ruuskanen and K. Tuominen, Nucl. Phys. B570, 379 (2000); X. Wang and M. Gyulassy, nucl-th/0008014.

[10] G. Calucci and D. Treleani, Phys. Rev. D49, 138 (1994); Phys. Rev. D50 (1994) 4703;

[11] G. Calucci and D. Treleani, Phys. Rev. D41, 3367 (1990);

[12] G. Calucci and D. Treleani, Phys. Rev. D44, 2746 (1991);

[13] K. J. Eskola, K. Kajantie and P. V. Ruuskanen, Eur. Phys. J. C1, 627 (1998);

[14] G. Calucci and D. Treleani, Proceedings of the workshop Hadron structure functions and parton distributions, Fermi National Accelerator Laboratory, World Scientific 1990; Int. J. Mod. Phys. A6, 4375 (1991);

[15] A. Bialas, M. Bleszynski and W. Czyz, Nucl. Phys. B111, 461 (1976);

[16] L. Ametller and D. Treleani, Int. J. Mod. Phys. A3, 521 (1988);

[17] K. J. Eskola, B. Muller and X. Wang, Phys. Lett. B374, 20 (1996);

[18] M. Gluck, E. Reya and A. Vogt, Eur. Phys. J. C5 (1998) 461;

[19] C. Albajar et al. [UA1 Collaboration], Nucl. Phys. B309, 405 (1988). 